TITLE:

\title{
Relaxation and diffusion in a globally coupled Hamiltonian system
}

AUTHOR(S):

Yamaguchi, Yoshiyuki Y

CITATION:

Yamaguchi, Yoshiyuki Y. Relaxation and diffusion in a globally coupled Hamiltonian system. Physical review. E, Statistical, nonlinear, and soft matter physics 2003, 68: 066210.

ISSUE DATE:

2003-12

URL:

http://hdl.handle.net/2433/202515

RIGHT:

C)2003 American Physical Society 


\title{
Construction of traveling clusters in the Hamiltonian mean-field model by nonequilibrium statistical mechanics and Bernstein-Greene-Kruskal waves
}

\author{
Yoshiyuki Y. Yamaguchi* \\ Department of Applied Mathematics and Physics, Graduate School of Informatics, Kyoto University, Kyoto 606-8501, Japan
}

(Received 23 January 2011; revised manuscript received 2 May 2011; published 18 July 2011)

\begin{abstract}
Traveling clusters are ubiquitously observed in the Hamiltonian mean-field model for a wide class of initial states, which are not predicted to become spatially inhomogeneous states by nonequilibrium statistical mechanics and by nonlinear Landau damping. To predict such a cluster state from a given initial state, we combine nonequilibrium statistical mechanics and a construction method of Bernstein-Greene-Kruskal (BGK) waves with the aid of phenomenological assumptions. The phenomenological theory is partially successful, and the theoretically constructed cluster states are in good agreement with $N$-body simulations. Robustness of the theory is also discussed for unsuccessful initial states.
\end{abstract}

DOI: 10.1103/PhysRevE.84.016211

PACS number(s): 05.45.-a, 05.20.Dd, 05.70.Ln, 52.35.Sb

\section{INTRODUCTION}

A Hamiltonian system with long-range interactions is frequently trapped in a quasistationary state or a quasiperiodic state before reaching thermal equilibrium. The lifetime of a trapped state diverges algebraically [1-3] as the number of particles increases, and hence observable states are such nonequilibrium states in the limit of large system size. A remarkable difference between the trapped state and thermal equilibrium is that the former depends on the initial values of nonconserved quantities; for instance, the order parameter. It is hence important to develop a theory which predicts a trapped state from a given initial state.

One such theory involves nonequilibrium statistical mechanics and was proposed by Lynden-Bell [4], and we refer to this theory as Lynden-Bell statistics. The theory is based on the description of $N$-body dynamics by the Vlasov equation [5]. Incompressibility of the Vlasov equation derives exclusivity of area elements of $\mu$ space and, accordingly, distribution functions of Fermi-Dirac type. The theory was originally introduced for self-gravitating systems, but was not fully successful in predicting the quasistationary states except for initial states which are close to the virial equilibrium $[6,7]$. See [8] for non-neutral plasmas.

Apart from the original objects, Lynden-Bell statistics are successfully applied to the so-called Hamiltonian mean-field (HMF) model with waterbag initial states [9]. The LyndenBell statistics draw a phase diagram of nonequilibrium phase transitions on the two-dimensional parameter plane spanned by the energy and initial order parameter axes, and the validity of the phase diagram is confirmed by $N$-body simulations [10]. A tricritical point is also discovered by Lynden-Bell statistics, while only the second-order phase transition occurs in thermal equilibrium.

Lynden-Bell statistics are useful to describe quasistationary states in the HMF model, but is not perfect. One failure is the existence of traveling clusters which are observed by $N$-body simulations over a wide part of the high-energy region

\footnotetext{
*yyama@i.kyoto-u.ac.jp
}

where Lynden-Bell statistics predict spatially homogeneous quasistationary states $[11,12]$. The failure of statistical prediction implies that the clusters are dynamically formed, and we need to combine statistical mechanics and dynamics to understand the mechanism of such traveling clusters. This combination can possibly develop a nonequilibrium statistical theory beyond Lynden-Bell statistics and is the main purpose of the present paper.

The state having traveling clusters is interpreted as a periodic solution to the Vlasov equation due to the periodic boundary condition of the HMF model. There are two dynamical mechanisms for constructing such a periodic state starting from a homogeneous background.

One of the mechanisms is nonlinear Landau damping [13], in which resonance between particles and waves is essential. Clusters result when the time scale of the linear Landau damping [14] is sufficiently long compared with the time scale in which resonant particles are trapped by waves. Such clusters are favorable to repulsive interactions; for instance, in plasmas, and can be observed in a wide class of initial states. Indeed, oscillations suggesting the existence of traveling clusters are observed experimentally $[15,16]$ and numerically [17]. Attractive interactions of the HMF model make it drastically more difficult to form such clusters. The parameter region is theoretically estimated where the nonlinear Landau damping produces the traveling clusters [18], and the region is very small compared with the region where the traveling clusters are observed by $N$-body simulations [12]. The nonlinear Landau damping is hence not suitable to explain the ubiquitous traveling clusters in the HMF model. Nonlinear Landau damping is based on perturbation techniques, and hence we need a nonperturbative method to understand the mechanism of the traveling clusters.

The other nonperturbative mechanism is an exact nonlinear traveling wave solution. The exact solution was discussed by Bohm and Gross [19], and formalized by Bernstein, Greene, and Kruskal [20]. The nonlinear traveling solution is hence known as a BGK wave. The basic idea of the BGK wave is to use the fact that any smooth functions of energy are stationary. Using this idea, a method is proposed to construct a nonlinear traveling solution from a homogeneous distribution [21,22]. 
Following, but modifying, this method, we theoretically construct a traveling cluster on a homogeneous background from a given initial waterbag state.

The construction method requires us to determine the velocities of the traveling clusters, and we determine them from the Landau dispersion relation. We choose the velocity as a frequency of the slowest decay mode, where frequency is equivalent to velocity in the HMF model due to the periodic boundary condition. We remark that, in the high-energy region, the homogeneous background is stable and no growing modes exist, and we have two of the slowest decay modes from symmetry of the homogeneous background. Consequently, we have to construct not one but two traveling clusters on a homogeneous background.

The strategy of constructing two traveling clusters is as follows: As a homogeneous background, we use a homogeneous quasistationary state which is predicted by Lynden-Bell statistics, since the statistics are successful except for the existence of the unpredicted clusters. Such a homogeneous state is the seed of a cluster state. One traveling cluster can be added onto the background by the method of Holloway and Dorning [21]. A two-cluster state can be constructed by nonlinear superposition of a pair of one-cluster states [23,24]. The constructed two-cluster state has unknown parameters, and we determine them by constraints of energy conservation, normalization conditions, and the self-consistency condition to ensure stationarity.

Construction of the present paper is as follows: The HMF model and Lynden-Bell statistics are introduced in Sec. II. The Landau dispersion relation is given in Sec. III. The one-cluster state and two-cluster state are constructed in Secs. IV and in $\mathrm{V}$, respectively. These states have unknown parameters, and they are determined in Sec. VI from constraints. The constructed two-cluster state is examined numerically in Sec. VII. The robustness of the theory is discussed in Sec. VIII. The conclusion appears in Sec. IX.

\section{MODEL AND LYNDEN-BELL DISTRIBUTION}

The HMF model [25,26] consists of $N$ particles confined on the unit circle and is expressed by the Hamiltonian

$$
H(\theta, p)=\sum_{j=1}^{N} \frac{p_{j}^{2}}{2}+\frac{1}{2 N} \sum_{j=1}^{N} \sum_{k=1}^{N}\left[1-\cos \left(\theta_{j}-\theta_{k}\right)\right],
$$

where $\theta_{j}$ is the angle of the $j$ th particle on the unit circle, and $p_{j}$ is the conjugate angular momentum. Interaction in the HMF model has the first Fourier component only, and hence the Landau dispersion relation is useful for wave number $k= \pm 1$ only. From the symmetry, we may set $k=1$ without loss of generality.

Despite the simplicity of interactions, the HMF model retains many qualitative properties of long-range interacting systems. The simplicity helps to compute the exact partition function [26], and to perform $N$-body simulations within a cost of $O(N)$ instead of $O\left(N^{2}\right)$, and hence it has been widely studied in the last decade.
The associated Vlasov equation to the HMF model is, for the one-particle distribution function $f$, written as

$$
\frac{\partial f}{\partial t}+\frac{\partial H_{1}}{\partial p} \frac{\partial f}{\partial \theta}-\frac{\partial H_{1}}{\partial \theta} \frac{\partial f}{\partial p}=0,
$$

where the one-particle Hamiltonian $H_{1}$ is

$$
H_{1}(\theta, p, t)=\frac{p^{2}}{2}-M_{x}[f](t) \cos \theta-M_{y}[f](t) \sin \theta .
$$

The vector $\left(M_{x}[f](t), M_{y}[f](t)\right)$ defined by

$$
\begin{aligned}
& M_{x}[f](t)=\int_{-\pi}^{\pi} d \theta \int_{-\infty}^{\infty} d p \cos \theta f(\theta, p, t), \\
& M_{y}[f](t)=\int_{-\pi}^{\pi} d \theta \int_{-\infty}^{\infty} d p \sin \theta f(\theta, p, t),
\end{aligned}
$$

is the order parameter, or the magnetization, of this system. Introducing the phase of the magnetization, denoted by $\phi(t)$, the one-particle Hamiltonian $H_{1}$ is rewritten as

$$
H_{1}(\theta, p, t)=\frac{p^{2}}{2}-M[f](t) \cos [\theta-\phi(t)],
$$

where

$$
M[f]=\sqrt{\left(M_{x}[f]\right)^{2}+\left(M_{y}[f]\right)^{2}} .
$$

A homogeneous stationary state $f$ implies $M[f]=0$, and $M$ takes the maximum value 1 when all the particles are at the same position.

We consider the waterbag initial distribution, $f_{\mathrm{WB}}(\theta, p)$, which is

$$
f_{\mathrm{WB}}(\theta, p)= \begin{cases}n_{0} & (\theta, p) \in R \\ 0, & \text { otherwise }\end{cases}
$$

where $R$ is a domain with finite area on $\mu$ space, and $\mu=$ $(-\pi, \pi] \times \mathbb{R}$. We set $R$ as a rectangular region:

$$
R=\left\{(\theta, p) \in \mu|| \theta\left|<\theta_{0},\right| p \mid<p_{0}\right\}
$$

From the normalization condition, $n_{0}=1 /|R|$ where $|R|$ is the area of $R$. The initial distribution (5) gives the initial magnetization pointing at the $x$ axis as

$$
\left(M_{x}\left[f_{\mathrm{WB}}\right], M_{y}\left[f_{\mathrm{WB}}\right]\right)=\left(M_{0}, 0\right),
$$

where $M_{0}=\left(\sin \theta_{0}\right) / \theta_{0}$. The family of initial waterbag distributions is parametrized by the pair $\left(\theta_{0}, p_{0}\right)$, or equivalently the pair of initial magnetization $M_{0}$ and energy

$$
U=\int_{-\pi}^{\pi} d \theta \int_{-\infty}^{\infty} d p\left(\frac{p^{2}}{2}+\frac{1-M[f] \cos \theta}{2}\right) f(\theta, p, t) .
$$

We remark that $U$ is conserved under the Vlasov equation (2), and the waterbag initial distribution (5) gives

$$
U=\frac{\left(p_{0}\right)^{2}}{6}+\frac{1-M_{0}^{2}}{2} .
$$



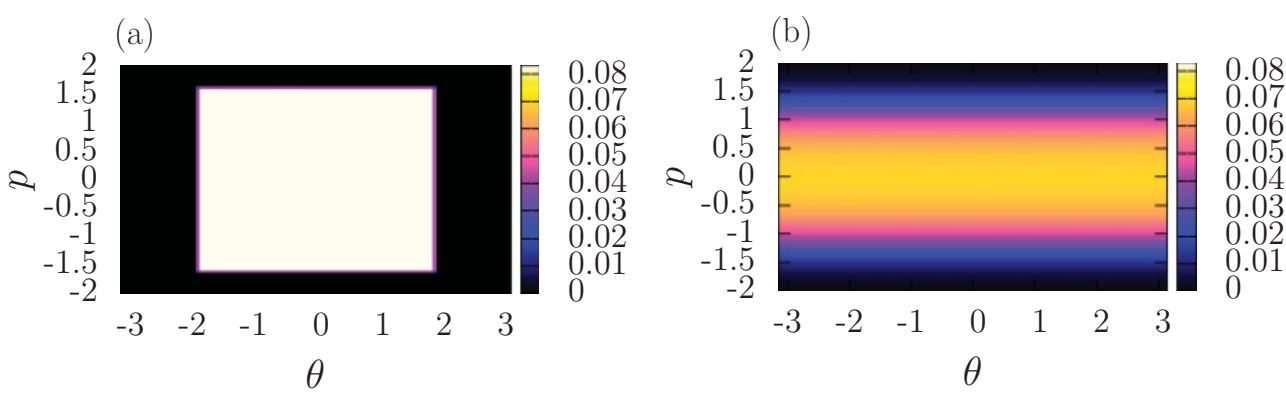

FIG. 1. (Color online) Waterbag initial distribution on $\mu$ space with $\left(M_{0}, U\right)=(0.5,0.8)$ (a) and corresponding homogeneous distribution predicted by Lynden-Bell statistics (b).

If the energy $U$ is high enough, Lynden-Bell statistics predict that a waterbag initial distribution goes to a spatially homogeneous distribution of

$$
f_{\mathrm{LB}}(p)=\frac{n_{0}}{e^{\alpha+\beta p^{2} / 2}+1},
$$

where the Lagrange multipliers $\alpha$ and $\beta$ are determined by substituting the Lynden-Bell distribution (9) into the normalization condition

$$
\int_{-\pi}^{\pi} d \theta \int_{-\infty}^{\infty} d p f_{\mathrm{LB}}(p)=1,
$$

and the energy constraint

$$
\int_{-\pi}^{\pi} d \theta \int_{-\infty}^{\infty} d p\left(\frac{p^{2}}{2}+\frac{1}{2}\right) f_{\mathrm{LB}}(p)=U .
$$

On the parameter plane $\left(M_{0}, U\right)$, the high-energy region is reported in [10] where the homogeneous Lynden-Bell distribution is stable. Roughly speaking, the lower boundary of the high-energy region is 0.75 for $M_{0}=1$, and it decreases as $M_{0}$ decreases. For instance, the point $\left(M_{0}, U\right)=(0.5,0.8)$ is in the high-energy region, and Lynden-Bell statistics gives a homogeneous distribution as shown in Fig. 1. Bachelard et al. reported that the traveling clusters appear in the high-energy region except for a neighborhood of the lower boundary [12]. Indeed, three clusters $\mathrm{A}, \mathrm{B}$, and $\mathrm{C}$ are observed in Fig. 2 by an $N$-body simulation for the point $\left(M_{0}, U\right)=(0.5,0.8)$. We neglect the cluster $\mathrm{B}$ since it has zero velocity and is not a traveling cluster, and construct the two traveling clusters A and $\mathrm{C}$ on the homogeneous background shown in Fig. 1(b).

\section{DISPERSION RELATION}

We decide the velocities of the two traveling clusters A and $\mathrm{C}$ from the Landau dispersion relation for the homogeneous distribution (9). Expanding the distribution around $f_{\mathrm{LB}}$ as

$$
f(\theta, p, t)=f_{\mathrm{LB}}(p)+f_{1}(\theta, p, t),
$$

where $f_{1}$ is a perturbation, we obtain the linearized Vlasov equation. The linearized equation is analyzed by performing the Fourier-Laplace transform of $f_{1}$ defined by

$$
\hat{f}_{1}(k, p, \omega)=\int_{-\pi}^{\pi} d \theta e^{-i k \theta} \int_{0}^{\infty} d t e^{i \omega t} f_{1}(\theta, p, t) .
$$

The dispersion relation is written by $D(\omega)=0$, where $D(\omega)$ is sometimes called the dispersion function and is expressed for wave number $k=1$ by

$$
D(\omega)=1+\pi \int_{L} \frac{f_{\mathrm{LB}}^{\prime}(p)}{p-\omega} d p .
$$

The integral path $L$ runs from $-\infty$ to $\infty$. The dispersion function is well-defined in the upper half $\omega$ plane to ensure convergence of the Laplace transform, and we need an analytic continuation to obtain $D(\omega)$ on the whole $\omega$ plane. The analytic continuation is introduced by Landau [14] and is performed by deforming the integral path $L$ on the complex $p$ plane to avoid the singular point $p=\omega$ when it crosses the real axis from the upper half plane to the lower. After the continuation, the Landau dispersion function is expressed as

$$
D(\omega)=1+\pi \mathrm{P} \int_{-\infty}^{\infty} \frac{f_{\mathrm{LB}}^{\prime}(p)}{p-\omega} d p+i 2 \pi^{2} \eta(\omega) f_{\mathrm{LB}}^{\prime}(\omega),
$$
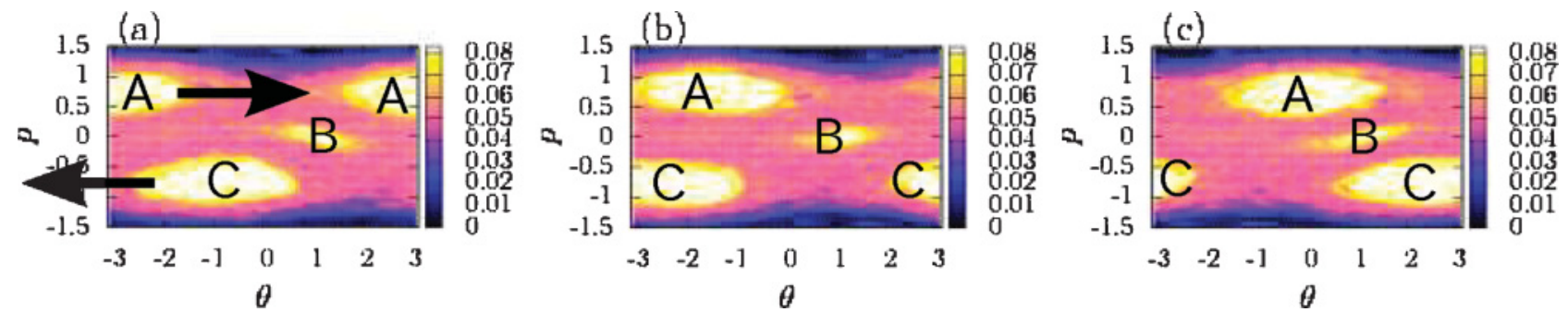

FIG. 2. (Color online) Temporal evolution of density plots in $\mu$ space. The density plots are taken at $t=500$ (a), 502 (b), and 504 (c). $\left(M_{0}, U\right)=(0.5,0.8)$ and $N=10^{6}$. Three clusters $\mathrm{A}, \mathrm{B}$, and $\mathrm{C}$ are observed. Clusters $\mathrm{A}$ and $\mathrm{C}$ propagate to the right and left, respectively, and cluster B does not propagate. 
where $\mathrm{P}$ denotes the principal value, and

$$
\eta(\omega)= \begin{cases}0, & \operatorname{Im} \omega>0 \\ 1 / 2, & \operatorname{Im} \omega=0 \\ 1, & \operatorname{Im} \omega<0 .\end{cases}
$$

The third term on the right-hand-side of (12) comes from the analytic continuation.

Temporal evolution of the perturbation $f_{1}$ is roughly proportional to $\exp (-i \omega t)$ by the inverse Laplace transform, where $\omega$ is a complex root of the Landau dispersion relation. The real and imaginary parts of $\omega$ hence express the frequency of oscillation and the growth rate, respectively. We assume that the homogeneous state (9) is stable, and hence the imaginary part is negative. We focus on the long-lasting slowest decay mode and denote the corresponding root by $\omega_{1}+i \omega_{2}$, whose imaginary part is largest. We call it the main root. We have a pair of main roots, since the distribution (9) is even and $\bar{D}(-\bar{\omega})=D(\omega)$ holds accordingly, where $\bar{\omega}$ is the complex conjugate of $\omega$. In other words, if $\omega_{1}+i \omega_{2}$ is a root of $D(\omega)$, then so is $-\omega_{1}+i \omega_{2}$. We assume $\omega_{1}>0$ without loss of generality. See [18,27] for the linear analysis in the HMF model.

\section{ONE-CLUSTER STATE}

We first construct a one-cluster state by following, but modifying, the procedure of Buchanan and Dorning [22]. The one-cluster state is constructed from the homogeneous Lynden-Bell distribution (9) and one of the main roots, $\omega_{1}+i \omega_{2}$, of the Landau dispersion function (12). We expect a cluster around the resonant point $p=\omega_{1}$.

As a preparation of the construction of a one-cluster state, we shift the cluster to the origin by the Galilei transform, $\tilde{p}=p-\omega_{1}$ and $\tilde{\theta}=\theta-\omega_{1} t$. The Vlasov equation (2) is transformed to

$$
\frac{\partial \tilde{f}}{\partial t}+\frac{\partial h_{1}}{\partial \tilde{p}} \frac{\partial \tilde{f}}{\partial \tilde{\theta}}-\frac{\partial h_{1}}{\partial \tilde{\theta}} \frac{\partial \tilde{f}}{\partial \tilde{p}}=0,
$$

where

$$
\tilde{f}(\tilde{\theta}, \tilde{p}, t)=f\left(\tilde{\theta}+\omega_{1} t, \tilde{p}+\omega_{1}, t\right)
$$

and

$$
h_{1}\left(\tilde{\theta}, \tilde{p} ; M_{1}\right)=\frac{\tilde{p}^{2}}{2}-M_{1} \cos \tilde{\theta} .
$$

The subscript 1 of $h_{1}$ and $M_{1}$ corresponds to the one-cluster state. The magnetization $M_{1}$ is defined by

$$
M_{1}=\int_{-\pi}^{\pi} d \tilde{\theta} \int_{-\infty}^{\infty} d \tilde{p} \cos \tilde{\theta} \tilde{f}(\tilde{\theta}, \tilde{p}, t) .
$$

We start from the homogeneous Lynden-Bell distribution

$$
\tilde{f}(\tilde{p})=f_{\mathrm{LB}}\left(\tilde{p}+\omega_{1}\right),
$$

which is obtained for a given pair of parameters $\left(M_{0}, U\right)$. What we have to do is to construct a one-cluster stationary state, since it becomes a periodic state by the inverse Galilei transform. The idea to construct a one-cluster stationary state from $\tilde{f}$ is to use the fact that any smooth distributions depending on $\tilde{\theta}$ and $\tilde{p}$ through $h_{1}\left(\tilde{\theta}, \tilde{p} ; M_{1}\right)$ are stationary if the magnetization (16) is determined self-consistently. One simple example is $\tilde{f}\left(h_{1}\left(\tilde{\theta}, \tilde{p} ; M_{1}\right)\right)$, but this type of distributions has three problems to be solved.

The first problem appears in the limit $M_{1} \rightarrow 0$. We require that the one-cluster state goes to the homogeneous state $\tilde{f}(\tilde{p})$ in this limit but $\tilde{f}\left(h_{1}\left(\tilde{\theta}, \tilde{p} ; M_{1}\right)\right)$ does not, and hence we consider

$$
\tilde{f}\left(\sqrt{2 h_{1}\left(\tilde{\theta}, \tilde{p} ; M_{1}\right)}\right)
$$

instead of the simple example. For this purpose, we omitted the integral constant in the one-particle Hamiltonian $h_{1}(15)$. The distribution (17) still has a problem in that it goes to $\tilde{f}(|\tilde{p}|)$ instead of $\tilde{f}(\tilde{p})$. We hence divide $\tilde{f}$ into even and odd parts, which are defined as

$$
\tilde{f}^{e}(\tilde{p})=\frac{\tilde{f}(\tilde{p})+\tilde{f}(-\tilde{p})}{2}, \quad \tilde{f}^{o}(\tilde{p})=\frac{\tilde{f}(\tilde{p})-\tilde{f}(-\tilde{p})}{2},
$$

respectively. Using this division, we introduce

$$
\begin{gathered}
G^{e}\left(\tilde{\theta}, \tilde{p} ; M_{1}\right)=\tilde{f}^{e}\left(\sqrt{2 h_{1}\left(\tilde{\theta}, \tilde{p} ; M_{1}\right)}\right), \\
G^{o}\left(\tilde{\theta}, \tilde{p} ; M_{1}\right) \begin{cases}\tilde{f}^{o}\left(\sqrt{2 h_{1}\left(\tilde{\theta}, \tilde{p} ; M_{1}\right)}\right) & \tilde{p} \geqslant 0 \\
-\tilde{f}^{o}\left(\sqrt{2 h_{1}\left(\tilde{\theta}, \tilde{p} ; M_{1}\right)}\right) & \tilde{p}<0,\end{cases}
\end{gathered}
$$

and then the distribution

$$
G^{e}\left(\tilde{\theta}, \tilde{p} ; M_{1}\right)+G^{o}\left(\tilde{\theta}, \tilde{p} ; M_{1}\right)
$$

depends on $(\tilde{\theta}, \tilde{p})$ only through $h_{1}\left(\tilde{\theta}, \tilde{p} ; M_{1}\right)$, and goes to $\tilde{f}(\tilde{p})$ in the limit $M_{1} \rightarrow 0$.

The second problem is that the argument $\sqrt{2 h_{1}}$ is not defined in the region $h_{1}<0$, which is the center part of the cluster, as shown in Fig. 3. To discuss clearly, we define positive- and negative-energy regions as

$$
C_{+}=\left\{(\tilde{\theta}, \tilde{p}) \mid h_{1}(\tilde{\theta}, \tilde{p}) \geqslant 0\right\}
$$

and

$$
C_{-}=\left\{(\tilde{\theta}, \tilde{p}) \mid h_{1}(\tilde{\theta}, \tilde{p})<0\right\},
$$

respectively. In previous works [21,22], the odd part $G^{o}$ is set to 0 in $C_{-}$, and we adopt this setting to make the onecluster state stationary. On the other hand, we introduce a different assumption from the previous works for the even

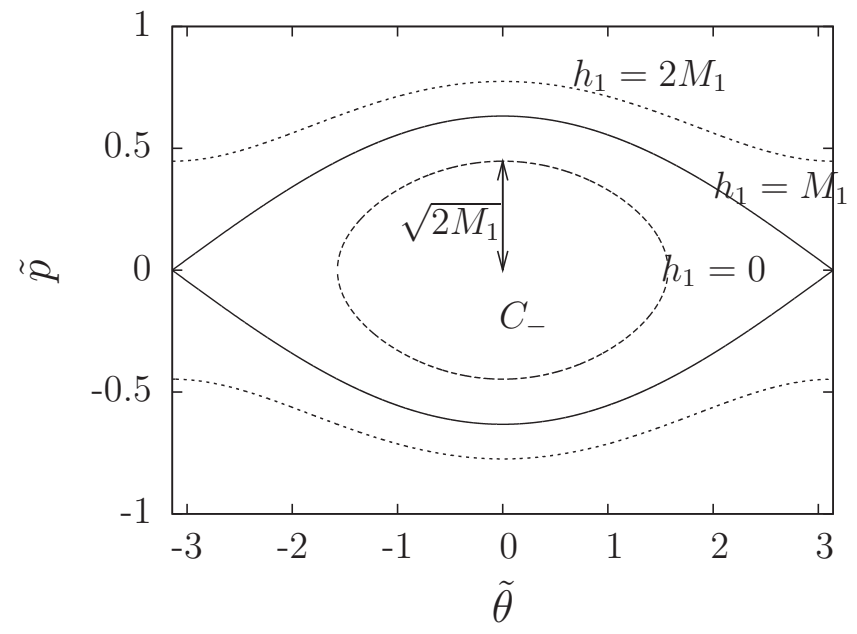

FIG. 3. Schematic of $\mu$ space around the Galilei-transformed cluster. 
part. Previously $G^{e}$ is expanded in the Taylor series of even terms in $C_{+}$. The expanded form has no square roots, and hence we may extend it to $C_{-}$. However, we observe that the height of the distribution function is almost constant in the clusters and is close to the initial height $n_{0}$ [see Figs. 1(a) and 2], and hence we phenomenologically assume that $G^{e}$ is constant in $C_{-}$. To this end, we redefine $G^{e}$ and $G^{o}$ on the whole $\mu$ space as

$$
\begin{gathered}
G^{e}(\tilde{\theta}, \tilde{p}):= \begin{cases}\tilde{f}^{e}\left(\sqrt{2 h_{1}(\tilde{\theta}, \tilde{p})}\right), & (\tilde{\theta}, \tilde{p}) \in C_{+} \\
n_{1}, & (\tilde{\theta}, \tilde{p}) \in C_{-}\end{cases} \\
G^{o}(\tilde{\theta}, \tilde{p}):= \begin{cases}\tilde{f}^{o}\left(\sqrt{2 h_{1}(\tilde{\theta}, \tilde{p})}\right), & (\tilde{\theta}, \tilde{p}) \in C_{+}, \tilde{p} \geqslant 0 \\
0, & (\tilde{\theta}, \tilde{p}) \in C_{-} \\
-\tilde{f}^{o}\left(\sqrt{2 h_{1}(\tilde{\theta}, \tilde{p})}\right), & (\tilde{\theta}, \tilde{p}) \in C_{+}, \tilde{p}<0,\end{cases}
\end{gathered}
$$

where $n_{1}$ is a positive constant. We remark that this phenomenological assumption might be reasonable if the domain $R$ (6) covers $C_{-}$. That is, $\theta_{0} \geqslant \pi / 2$ and $p_{0}>\omega_{1}+\sqrt{2 M_{1}}$, or equivalently

$$
M_{0} \leqslant \frac{2}{\pi} \text { and } U \geqslant \frac{\left(\omega_{1}+\sqrt{2 M_{1}}\right)^{2}}{6}+\frac{1-M_{0}^{2}}{2} .
$$

The third problem is that the summation $G^{e}+G^{o}$ is not stationary, since there is an odd part in the region $0<$ $h_{1}(\tilde{\theta}, \tilde{p})<M_{1}$. In addition, $G^{e}+G^{o}$ is discontinuous on the line $h_{1}\left(\tilde{\theta}, \tilde{p} ; M_{1}\right)=0$ unless $\tilde{f}^{e}(0)=n_{1}$ and $\tilde{f}^{o}(0)=0$. We therefore introduce a smoothing function

$$
S(\eta)= \begin{cases}0, & \eta \leqslant 1 \\ \frac{1}{2}\left[1+\tanh \frac{3-2 \eta}{2(\eta-1)(\eta-2)}\right], & 1<\eta<2 \\ 1, & \eta \geqslant 2\end{cases}
$$

and cut such unsuitable parts of the distribution. We remark that the choice of a smoothing function is arbitrary and is the unique ambiguity of the present theory. Using the smoothing function we modify the even part as

$$
G^{e}(\tilde{\theta}, \tilde{p})+\left[n_{1}-G^{e}(\tilde{\theta}, \tilde{p})\right] S\left(2-h_{1}(\tilde{\theta}, \tilde{p}) / M_{1}\right),
$$

and the odd part as

$$
G^{o}(\tilde{\theta}, \tilde{p}) S\left(h_{1}(\tilde{\theta}, \tilde{p}) / M_{1}\right) .
$$

The first modification smoothly connects the cluster part to the high-energy region in the intermediate region $0<$ $h_{1}\left(\tilde{\theta}, \tilde{p} ; M_{1}\right)<M_{1}$. The second modification makes the odd part vanish inside the separatrix, $h_{1}\left(\tilde{\theta}, \tilde{p} ; M_{1}\right) \leqslant M_{1}$ and smoothly connects the low-energy region to the high-energy region in $M_{1}<h_{1}\left(\tilde{\theta}, \tilde{p} ; M_{1}\right)<2 M_{1}$.

The constructed one-cluster stationary state is expressed as

$$
\begin{aligned}
\tilde{f}_{1+}(\tilde{\theta}, \tilde{p})= & \mathcal{N}_{1}\left(G^{e}(\tilde{\theta}, \tilde{p})\left\{1-S\left(2-h_{1}\left(\tilde{\theta}, \tilde{p} ; M_{1}\right) / M_{1}\right)\right\}\right. \\
& \left.+G^{o}(\tilde{\theta}, \tilde{p}) S\left(h_{1}(\tilde{\theta}, \tilde{p}) / M_{1}\right)\right) \\
& +\gamma_{1} n_{0} S\left(2-h_{1}(\tilde{\theta}, \tilde{p}) / M_{1}\right),
\end{aligned}
$$

where the new variable $\gamma_{1}=\mathcal{N}_{1} n_{1} / n_{0}$ represents the remained ratio of distribution in the center of cluster $C_{-}$from the initial value $n_{0}$, and $\mathcal{N}_{1}$ is the normalization factor depending on $M_{1}$ and $\gamma_{1}$. The unknown parameters will be determined from a given $U$ and $M_{0}$ in Sec. VI. The subscript $1+$ of $\tilde{f}$ represents the existence of one cluster on the positive $p$ side. By the inverse Galilei transform of $\tilde{f}_{1+}$, we obtain a periodic solution $f_{1+}$ with one cluster around $p=\omega_{1}$ as

$$
f_{1+}(\theta, p, t)=\tilde{f}_{1+}\left(\theta-\omega_{1} t, p-\omega_{1}\right) .
$$

The state $\tilde{f}_{1-}(\tilde{\theta}, \tilde{p})$, which has one cluster around $p=-\omega_{1}$, is also constructed in the same manner by replacing the definitions of $\tilde{\theta}$ and $\tilde{p}$ by $\tilde{\theta}=\theta+\omega_{1} t$ and $\tilde{p}=p+\omega_{1}$, respectively. The Galilei-transformed distribution

$$
f_{1-}(\theta, p, t)=\tilde{f}_{1-}\left(\theta+\omega_{1} t, p+\omega_{1}\right)
$$

is straightforwardly obtained by using the same values of $M_{1}, \mathcal{N}_{1}$, and $\gamma_{1}$ with ones for $f_{1+}$ from symmetry.

\section{TWO-CLUSTER STATE}

A pair of one-cluster states can be nonlinearly superposed by following the procedure of Buchanan-Dorning [23] if the clusters are small enough. Let us define $\tilde{p}_{ \pm}=p \mp \omega_{1}$ and $\tilde{\theta}_{ \pm}=\theta \mp \omega_{1} t$, and redefine the one-particle Hamiltonian as

$$
h_{2 \pm}\left(\tilde{\theta}_{ \pm}, \tilde{p}_{ \pm}\right)=\frac{\tilde{p}_{ \pm}^{2}}{2}-M_{1} \cos \tilde{\theta}_{ \pm}-M_{1} \frac{\tilde{p}_{ \pm}}{\tilde{p}_{ \pm} \pm 2 \omega_{1}} \cos \tilde{\theta}_{\mp} .
$$

The Hamiltonians $h_{2 \pm}$ are integrals up to $O\left(M_{1}\right)$; in other words, by neglecting $O\left(M_{1}^{2}\right)$ in the time-dependent Hamiltonian system

$$
H_{2}=\frac{p^{2}}{2}-M_{1} \cos \left(\theta-\omega_{1} t\right)-M_{1} \cos \left(\theta+\omega_{1} t\right),
$$

which has two clusters of magnetization $M_{1}$ around $p=\omega_{1}$ and $p=-\omega_{1}$.

Replacing $h_{1}$ by $h_{2 \pm}$, we can reconstruct the one-cluster state, and the reconstructed one-cluster states are denoted $f_{2 \pm}$. The Galilei-transformed one-cluster states $\tilde{f}_{2 \pm}$ are represented by

$$
\begin{aligned}
\tilde{f}_{2 \pm}(\tilde{\theta}, \tilde{p})= & \mathcal{N}_{2 \pm}\left(G^{e}(\tilde{\theta}, \tilde{p})\left\{1-S\left(2-h_{2 \pm}(\tilde{\theta}, \tilde{p}) / M_{1}\right)\right\}\right. \\
& \left.\left.+G^{o}(\tilde{\theta}, \tilde{p}) S\left(h_{2 \pm}(\tilde{\theta}, \tilde{p}) / M_{1}\right)\right\}\right) \\
& +\gamma_{2} n_{0} S\left(2-h_{2 \pm}(\tilde{\theta}, \tilde{p}) / M_{1}\right) .
\end{aligned}
$$

Thanks to the symmetry of the Hamiltonian,

$$
h_{2-}(-\theta,-p)=h_{2+}(\theta, p),
$$

the distribution has similar symmetry:

$$
\tilde{f}_{2-}(-\theta,-p)=\tilde{f}_{2+}(\theta, p) .
$$

Consequently, the normalization factors $\mathcal{N}_{2 \pm}$ are identical (i.e., $\mathcal{N}_{2+}=\mathcal{N}_{2-}=\mathcal{N}_{2}$ ). The nonlinear superposition between $f_{2+}$ and $f_{2-}$, which is a two-cluster state, is expressed by

$$
f_{2}(\theta, p, t)= \begin{cases}f_{2+}(\theta, p, t), & p \geqslant 0 \\ f_{2-}(\theta, p, t), & p<0\end{cases}
$$

\section{DETERMINATION OF PARAMETERS}

Looking at one-cluster and two-cluster states, (20) and (24), respectively, we find that there are five undetermined parameters: $M_{1}, \mathcal{N}_{1}, \mathcal{N}_{2}, \gamma_{1}$, and $\gamma_{2}$. To reduce the number 

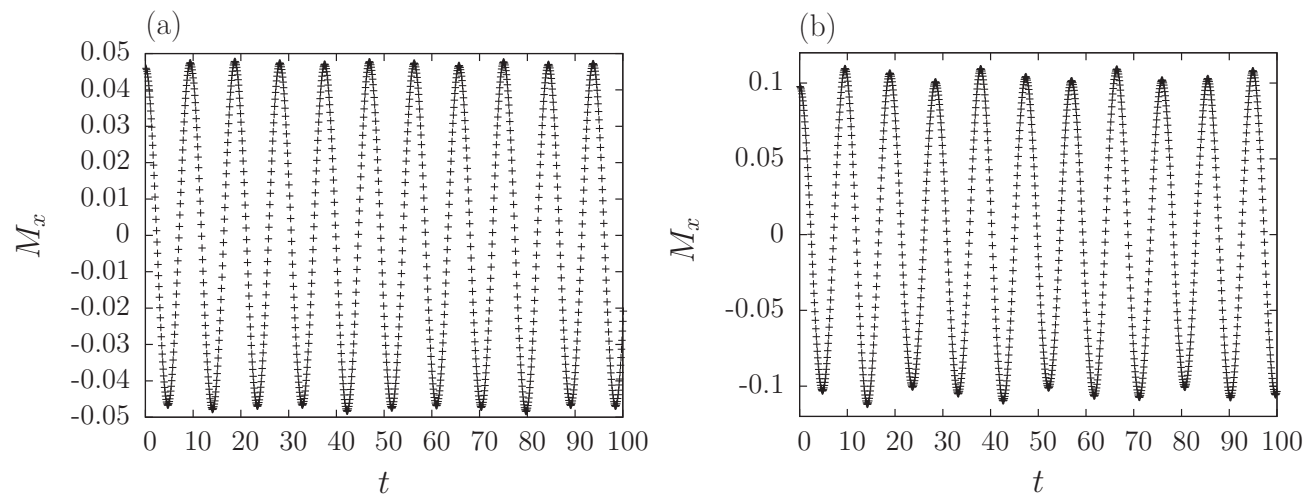

FIG. 4. Temporal evolutions of $M_{x}(t)$ in $N$-body simulations for $\left(M_{0}, U\right)=(0.5,0.8)$ with $N=10^{7}$. Initial states are (a) $f_{1+}$ and (b) $f_{2}$.

of unknown parameters, we assume that $\gamma_{1}=\gamma_{2}=\gamma$. This assumption says that the distribution takes the same value in the cluster center both for one- and two-cluster states.

The four unknown parameters are determined by solving the following four conditions: The self-consistent equation for $M_{1}$,

$$
\int_{-\pi}^{\pi} d \theta \int_{-\infty}^{\infty} d p \cos \theta f_{1 \pm}(\theta, p, t)=M_{1},
$$

the normalization condition for $f_{1 \pm}$,

$$
\int_{-\pi}^{\pi} d \theta \int_{-\infty}^{\infty} d p f_{1 \pm}(\theta, p, t)=1,
$$

the normalization condition for $f_{2}$,

$$
\int_{-\pi}^{\pi} d \theta \int_{-\infty}^{\infty} d p f_{2}(\theta, p, t)=1
$$

and energy condition for $f_{2}$,

$$
\begin{aligned}
\int_{-\pi}^{\pi} d \theta \int_{-\infty}^{\infty} d p \frac{p^{2}}{2} f_{2}(\theta, p, t)+\frac{1}{2} \\
-\frac{1}{2}\left(\int_{-\pi}^{\pi} d \theta \int_{-\infty}^{\infty} d p \cos \theta f_{2}(\theta, p, t)\right)^{2}=U,
\end{aligned}
$$

where $U$ is computed from a given waterbag initial state by (8). In each of (25) and (26) we may choose one of $f_{1+}$ and $f_{1-}$ since the two states give equivalent conditions. We remark that $M_{1}$ must be determined self-consistently with $f_{1 \pm}$ instead of $f_{2 \pm}$ to ensure stationarity of $\tilde{f}_{1 \pm}$. Using stationarity of $\tilde{f}_{1 \pm}$ (in other words, the periodicity of $f_{1 \pm}$ ), the nonlinear superposition performed in Sec. V makes $f_{2}$ approximately periodic. The energy of $f_{1 \pm}$ can be also considered, but the value must be different from that appearing in (28) and is hence not useful to determine the unknown parameters. Details of a computational method for the four unknown parameters are described in Appendix.

\section{NUMERICAL TEST}

In this section we fix the parameters characterizing a waterbag initial distribution as $U=0.8$ and $M_{0}=0.5$. We remark that this waterbag initial condition does not yield clusters by nonlinear Landau damping [18]. Another remark is that this pair of parameters satisfies condition (18) since $\omega_{1} \simeq$ 0.67 and $M_{1} \simeq 0.045$. We examined whether the constructed one- and two-cluster states are suitable as initial states of periodic solutions, and compared the theoretically constructed distribution with that computed by an $N$-body simulation.

To confirm the periodicity, we randomly draw $N$ pairs of $\left(\theta_{i}, p_{i}\right)$ from the one-cluster state $f_{1+}$, and set them as initial conditions of an $N$-body simulation. The numerical integrations of the canonical equations of motion are performed by the fourth-order symplectic integrator with time slice 0.1 . Another simulation is also performed for the two-cluster state $f_{2}$. Evolutions of $M_{x}(t)$ are reported in Fig. 4. Both one- and
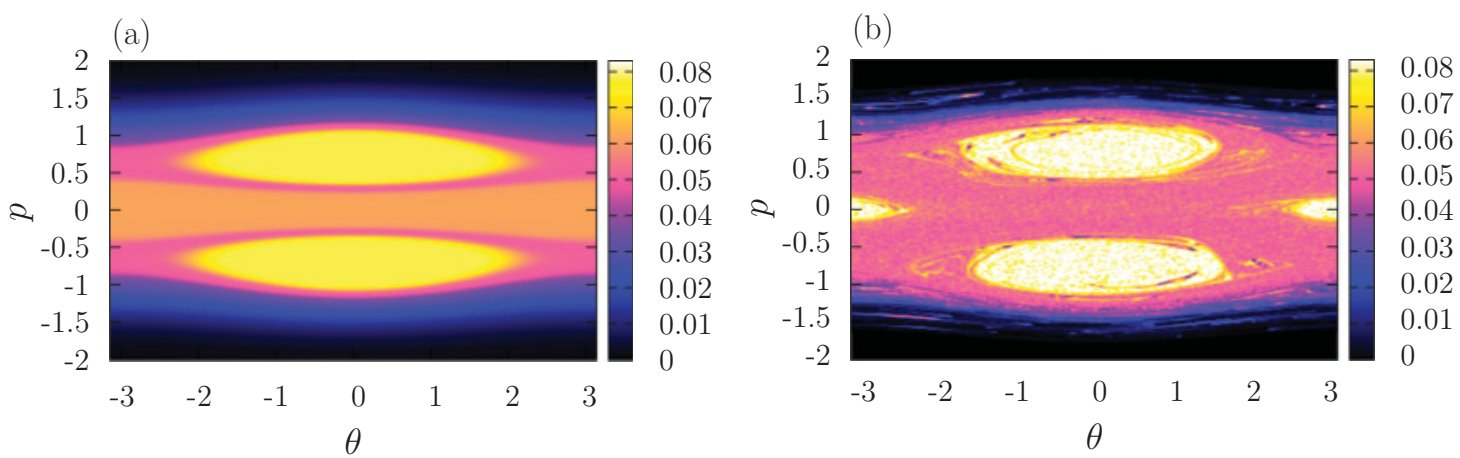

FIG. 5. (Color online) Density plots of distributions in $\mu$ space. $\left(M_{0}, U\right)=(0.5,0.8)$. (a) Theory $f_{2}$. (b) $N$-body simulation from waterbag initial state with $N=10^{7}$ at $t=507.1$. 


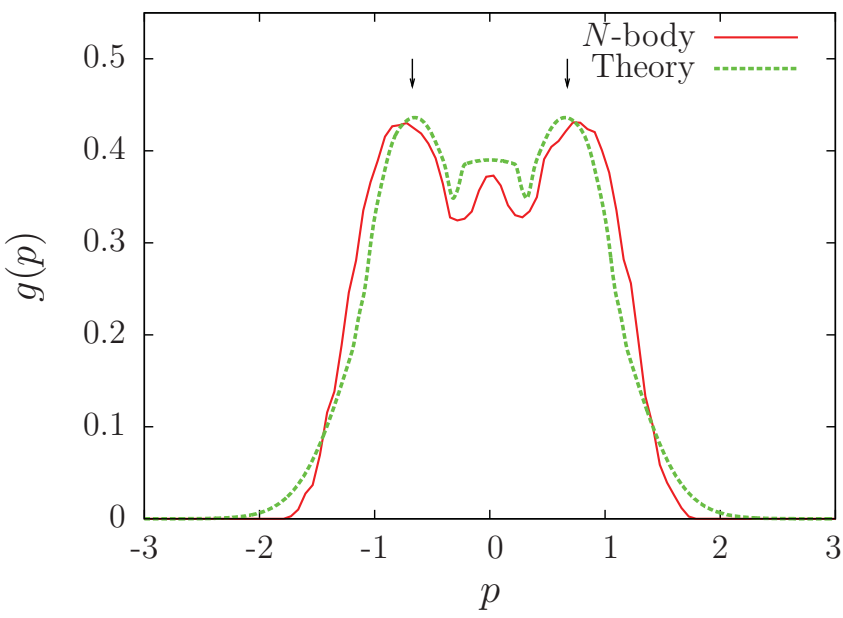

FIG. 6. (Color online) Momentum distributions computed from Fig. 5. $\left(M_{0}, U\right)=(0.5,0.8)$. $N$-body simulation with $N=10^{7}$ at $t=507.1$ (solid red line), and the theoretically obtained $f_{2}$ (dashed green line). The arrows mark positions $\pm \omega_{1}$ of the main roots.

two-cluster states are almost periodic, although the former is better than the latter. Slight breaking of periodicity for the latter may be caused by the approximation introduced in the nonlinear superposition.

Comparison between theory and an $N$-body simulation is demonstrated by observing density plots on $\mu$ space and momentum distributions. The density plots are exhibited in Fig. 5, which are the theoretically constructed $f_{2}$ and the one computed by an $N$-body simulation with the waterbag initial state. The latter is taken as a snap shot at the time $t=507.1$ to set the phases of two clusters' zeros for comparison. The two density plots are in good agreement with each other, except for the existence of the small cluster around $p=0$ in the $N$-body simulation, which corresponds to the neglected cluster $\mathrm{B}$ in Fig. 2. We demonstrate further comparison by observing momentum distributions, defined by

$$
g(p)=\int_{-\pi}^{\pi} f(\theta, p) d \theta .
$$

The momentum distributions are computed from the density plots representing $f(\theta, p)$ shown in Fig. 5, and agreement between them is shown in Fig. 6.

\section{ROBUSTNESS OF THEORY}

The theoretical distribution is in good agreement with the $N$-body simulation for $\left(M_{0}, U\right)=(0.5,0.8)$, but the theory does not always work well. For instance, the parameter pair $\left(M_{0}, U\right)=(0.5,0.7)$ has no solutions to the four conditions (25)-(28).

For checking the robustness of the theory, we introduce an artificial energy $U_{\text {art }}$ and replace it with the proper $U$ in the energy condition (28). The ranges of $U_{\text {art }}$ for which solutions are found are arranged in Table I. Momentum distributions for some $U_{\text {art }}$ picked up from such ranges are shown in Fig. 7 for $U=0.7$ and for $U=0.9$ with $M_{0}=0.5$. The theory with a suitable choice of $U_{\text {art }}$ gives a good approximation to the $N$-body result; for instance, $U_{\text {art }}=0.691$ for $U=0.7$ and
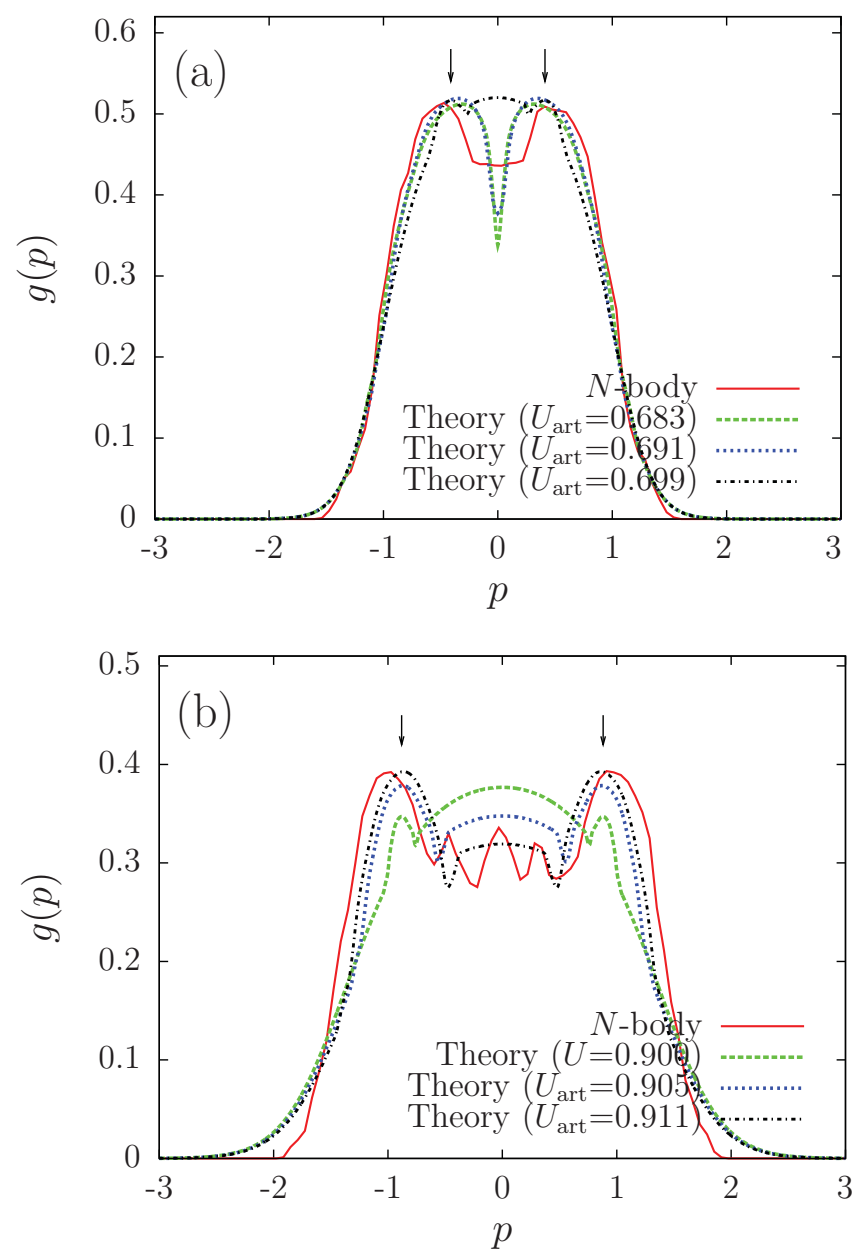

FIG. 7. (Color online) Momentum distributions for $\left(M_{0}, U\right)=$ $(0.5,0.7)$ (a) and for $\left(M_{0}, U\right)=(0.5,0.9)(\mathrm{b})$. Solid red distributions are obtained by $N$-body simulations with $N=10^{7}$ at $t=509.5$ for (a) and at $t=502.1$ for (b). They are taken when the phases of two clusters coincide. Theoretical distributions are reported for some values of artificial energy $U_{\text {art }}$, where $U_{\text {art }}$ increases in the order of dashed green, dotted blue, and dot-dashed black. The arrows mark positions $\pm \omega_{1}$ of the main roots.

$U_{\text {art }}=0.911$ for $U=0.9$. Discrepancies between the proper $U$ and the suitable $U_{\text {art }}$ are less than $1.3 \%$, and hence we need a more accurate theory, both for the based homogeneous backgrounds and for cluster states, in order to make the theory robust.

Another check of the robustness of the theory regards the ambiguity included in the theory, which is the choice of the smoothing function (19). We remark that a change of choice, for instance $S(\eta)$ connecting 0 and 1 in the interval

TABLE I. Ranges of $U_{\text {art }}$ in which solutions to the four conditions are found.

\begin{tabular}{lc}
\hline \hline$U$ & Range of $U_{\text {art }}$ \\
\hline 0.9 & $0.900 \leqslant U_{\text {art }} \leqslant 0.911$ \\
0.8 & $0.800 \leqslant U_{\text {art }} \leqslant 0.800$ \\
0.7 & $0.683 \leqslant U_{\text {art }} \leqslant 0.699$ \\
\hline \hline
\end{tabular}


$[1,1.5]$ instead of $[1,2]$, does not qualitatively affect the above robustness and distributions with $U_{\text {art }}$.

\section{CONCLUSION}

A phenomenological theory has been proposed for predicting a two-cluster state from a given waterbag initial state, which is characterized by two parameters. The twocluster states are constructed on homogeneous backgrounds predicted by the Lynden-Bell statistics, and include four unknown parameters. Determining the four parameters by four constraints, the two-cluster states are in good agreement with $N$-body simulations starting from waterbag initial states at some points on the parameter plane.

However, the theoretical approximations possibly do not agree with $N$-body simulations. Moreover, no solutions are obtained to the four constraints in a region of the parameter plane. This nonrobustness of the theory has been examined by replacing the proper energy $U$, appearing in one of the four constraints, with an artificial energy $U_{\text {art }}$. The range of $U_{\text {art }}$ giving the solutions is very narrow, and the narrow window suggests that we need a more accurate prediction of the homogeneous background beyond Lynden-Bell statistics. For instance, in the tail of momentum distributions, $N$-body simulations give sharper decay than that predicted by LyndenBell statistics, and hence the energy effectively changes from the proper value $U$.

Nevertheless, since the two-cluster states with the artificial energy are close to the $N$-body simulations, we may conjecture that the mechanism of the traveling clusters on a homogeneous background starting from a waterbag initial state is as follows: A waterbag initial state with high energy goes toward a homogeneous Lynden-Bell state, but does not perfectly coincide. Some particles are trapped in the resonant region which is predicted by the Landau dispersion relation, and the state reaches an almost periodic state with traveling clusters. Such a cluster state can be approximately constructed as a nonlinearly superposed BGK wave.

The above scenario makes it possible to discuss the following two phenomena observed in the HMF model: One phenomenon is the bifurcation from one big cluster to the two small traveling clusters reported in [12]. The bifurcation point corresponds to the critical point of the order-disorder transition in nonequilibrium. Velocities of the two small traveling clusters are understood by the real part of the main roots, and hence we can theoretically reproduce the bifurcation diagram from the Landau dispersion relation, although it is not reported. The other phenomenon is the long-lasting traveling clusters on an inhomogeneous background [28]. The clusters appear around the separatrix, and the position of the clusters can be understood by the dispersion relation. To consider the resonance frequency on an inhomogeneous background, we need to introduce the action-angle variables and define frequency as the derivative of the one-particle Hamiltonian with respect to action. The frequency has a sharp slope around the separatrix action, and a wide range of frequency is hence expected around the separatrix. Appearance of the traveling clusters in resonance is hence favorable around the separatrix. The dispersion relation for the inhomogeneous background is complicated [29,30] and reconstruction of the traveling clusters along this scenario is left as a future problem.

The strategy of the present theory is useful in other systems, but we need (i) to extend the construction method of BGK waves to inhomogeneous backgrounds and (ii) to use a reasonable nonequilibrium statistical theory to describe quasistationary states. For instance, the core-halo structure found in self-gravitating systems and plasma systems cannot be described by the Lynden-Bell theory. Recently, other approaches to describe quasistationary states have been proposed by reproducing the core-halo structure [31] and by assuming conservation of energy for each particle [3]. It might be interesting to replace Lynden-Bell statistics in the present theory with one of these approaches for the HMF model and for other realistic models. Another improvement of the present theory is possible for the construction of periodic states, since we considered two traveling clusters and neglected the other smaller clusters. This improvement increases the number of unknown parameters; for instance, the height of distribution in each cluster, and we have to introduce more constraints in order to determine such parameters. The Vlasov equation has an infinite number of Casimir invariants, and using the Casimirs as the additional constraints might be a possible way.

\section{ACKNOWLEDGMENTS}

The author expresses his thanks to J.Barré for introducing him the procedure to construct a one-cluster stationary state and nonlinear superposition. This work is supported by the Ministry of Educations, Science, Sports and Culture, Grantin-Aid for Young Scientists (B), 19760052.

\section{APPENDIX: COMPUTATION OF PARAMETERS}

The unknown parameters $\mathcal{N}_{2}, \gamma, \mathcal{N}_{1}$, and $M_{1}$ are computed from the following four steps: (i) fixing a value of $M_{1}$, (ii) obtaining $\mathcal{N}_{2}$ and $\gamma$ as functions of $M_{1}$ by Eqs. (27) and (28), (iii) computing $\mathcal{N}_{1}$ as a function of $M_{1}$ by Eq. (26), and (iv) determining $M_{1}$ self-consistently by Eq. (25).

In step (ii), we use the fact that the integrals of Eqs. (27) and (28) can be represented by using $f_{2+}$ from the symmetry of $f_{2-}(-\theta,-p)=f_{2+}(\theta, p)$. The function $f_{2+}$ is, referring to Eq. (24), written in the form

$$
f_{2+}(\theta, p)=\mathcal{N}_{2} A_{2}(\theta, p)+\gamma B_{2}(\theta, p) .
$$

The two conditions (27) and (28) are hence rewritten as

$$
1=2\left[\mathcal{N}_{2}\left\langle A_{2}\right\rangle+\gamma\left\langle B_{2}\right\rangle\right]
$$

and

$$
\begin{aligned}
U-\frac{1}{2}= & \mathcal{N}_{2}\left\langle A_{2} p^{2}\right\rangle+\gamma\left\langle B_{2} p^{2}\right\rangle-2\left(\mathcal{N}_{2}\left\langle A_{2} \cos \theta\right\rangle\right. \\
& \left.+\gamma\left\langle B_{2} \cos \theta\right\rangle\right)^{2}
\end{aligned}
$$

where, for a function $A$ of $(\theta, p)$, the symbol $\langle\cdot\rangle$ represents

$$
\langle A\rangle=\int_{-\pi}^{\pi} d \theta \int_{0}^{\infty} d p A(\theta, p) .
$$

Note that the integral starts from 0 instead of $-\infty$ in the $p$ integration. The averaged values can be numerically computed, 
and the solutions of Eqs. (A2) and (A3) with respect to $\mathcal{N}_{2}$ and $\gamma$ are computed as

$$
\mathcal{N}_{2}=\frac{-b \pm \sqrt{b^{2}-4 a c}}{2 a}
$$

and

$$
\gamma=\frac{1}{\left\langle B_{2}\right\rangle}\left[\frac{1}{2}-\mathcal{N}_{2}\left\langle A_{2}\right\rangle\right],
$$

where the coefficients $a, b$, and $c$ are

$$
\begin{aligned}
a= & {\left[\left\langle B_{2}\right\rangle\left\langle A_{2} \cos \theta\right\rangle-\left\langle A_{2}\right\rangle\left\langle B_{2} \cos \theta\right\rangle\right]^{2}, } \\
b= & \frac{1}{2}\left\langle B_{2}\right\rangle\left[\left\langle A_{2}\right\rangle\left\langle B_{2} p^{2}\right\rangle-\left\langle B_{2}\right\rangle\left\langle A_{2} p^{2}\right\rangle\right] \\
& +\left\langle B_{2} \cos \theta\right\rangle\left[\left\langle B_{2}\right\rangle\left\langle A_{2} \cos \theta\right\rangle-\left\langle A_{2}\right\rangle\left\langle B_{2} \cos \theta\right\rangle\right], \\
c= & \frac{1}{4}\left[-\left\langle B_{2}\right\rangle\left\langle B_{2} p^{2}\right\rangle+\left\langle B_{2} \cos \theta\right\rangle^{2}+(2 U-1)\left\langle B_{2}\right\rangle^{2}\right] .
\end{aligned}
$$

From two pairs of solutions, we adopt a pair satisfying $\mathcal{N}_{2}>0$ and $0 \leqslant \gamma \leqslant 1$. We remark that only one pair was acceptable at most in our computations shown in the text.

For step (iii), we also rewrite $f_{1+}$ in the form

$$
f_{1+}(\theta, p)=\mathcal{N}_{1} A_{1}(\theta, p)+\gamma B_{1}(\theta, p) .
$$

From the normalization condition (26), we get

$$
\mathcal{N}_{1}=\frac{1-\gamma \int_{-\pi}^{\pi} d \theta \int_{-\infty}^{\infty} d p B_{1}}{\int_{-\pi}^{\pi} d \theta \int_{-\infty}^{\infty} d p A_{1}} .
$$

Note that the lower boundary is $-\infty$ in the $p$ integration.

Finally we seek $M_{1}$ which satisfies the self-consistent equation (25).
[1] Y. Y. Yamaguchi, J. Barré, F. Bouchet, T. Dauxois, and S. Ruffo, Physica A 337, 36 (2004).

[2] L. G. Moyano and C. Anteneodo, Phys. Rev. E 74, 021118 (2006).

[3] P. de Buyl, D. Mukamel, and S. Ruffo, e-print arXiv:1012.2594.

[4] D. Lynden Bell, Mon. Not. R. Astron. Soc. 136, 101 (1967).

[5] W. Braun and K. Hepp, Commun. Math. Phys. 56, 101 (1977).

[6] Y. Y. Yamaguchi, Phys. Rev. E 78, 041114 (2008).

[7] Y. Levin, R. Pakter, and F. B. Rizzato, Phys. Rev. E 78, 021130 (2008).

[8] Y. Levin, R. Pakter, and T. N. Teles, Phys. Rev. Lett. 100, 040604 (2008).

[9] A. Antoniazzi, D. Fanelli, J. Barré, P.-H. Chavanis, T. Dauxois, and S. Ruffo, Phys. Rev. E 75, 011112 (2007).

[10] A. Antoniazzi, D. Fanelli, S. Ruffo, and Y. Y. Yamaguchi, Phys. Rev. Lett. 99, 040601 (2007).

[11] A. Antoniazzi, F. Califano, D. Fanelli, and S. Ruffo, Phys. Rev. Lett. 98, 150602 (2007).

[12] R. Bachelard, C. Chandre, D. Fanelli, X. Leoncini, and S. Ruffo, Phys. Rev. Lett. 101, 260603 (2008).

[13] T. O’Neil, Phys. Fluids 8, 2255 (1965).

[14] L. Landau, J. Phys. USSR 10, 25 (1946).
[15] J. H. Malmberg and C. B. Wharton, Phys. Rev. Lett. 19, 775 (1967).

[16] R. N. Franklin, S. M. Hamberger, and G. J. Smith, Phys. Rev. Lett. 29, 914 (1972).

[17] G. Manfredi, Phys. Rev. Lett. 79, 2815 (1997).

[18] J. Barré and Y. Y. Yamaguchi, Phys. Rev. E 79, 036208 (2009).

[19] D. Bohm and E. P. Gross, Phys. Rev. 75, 1851 (1949).

[20] I. B. Bernstein, J. M. Greene, and M. D. Kruskal, Phys. Rev. 108, 546 (1957).

[21] J. P. Holloway and J. J. Dorning, Phys. Rev. A 44, 3856 (1991).

[22] M. Buchanan and J. Dorning, Phys. Rev. E 52, 3015 (1995).

[23] M. Buchanan and J. J. Dorning, Phys. Rev. Lett. 70, 3732 (1993).

[24] M. Buchanan and J. Dorning, Phys. Rev. E 50, 1465 (1994).

[25] S. Inagaki and T. Konishi, Publ. Astron. Soc. Jpn. 45, 733 (1993).

[26] M. Antoni and S. Ruffo, Phys. Rev. E 52, 2361 (1995).

[27] A. Campa, T. Dauxois, and S. Ruffo, Phys. Rep. 480, 57 (2009).

[28] H. Morita and K. Kaneko, Phys. Rev. Lett. 96, 050602 (2006).

[29] P. H. Chavanis, Physica A 377, 469 (2007).

[30] J. Barré, A. Olivetti, and Y. Y. Yamaguchi, J. Stat. Mech. (2010) P08002.

[31] R. Pakter and Y. Levin, Phys. Rev. Lett. 106, 200603 (2011). 